\title{
On the friction and adhesion hysteresis between polymer brushes attached to curved surfaces: Rate and solvation effects
}

\author{
Sissi de BEER ${ }^{1,2}$, G. Djuidjé KENMOÉ ${ }^{3}$, Martin H. MÜSER ${ }^{2, *}$ \\ ${ }^{1}$ Materials Science and Technology of Polymers, MESA+ Institute for Nanotechnology, University of Twente, P. O. Box 217, 7500 AE \\ Enschede, the Netherlands \\ ${ }^{2}$ Jülich Supercomputing Centre Institute for Advanced Simulation FZ Jülich, 52425 Jülich, Germany \\ ${ }^{3}$ Department of Physics, Laboratory of Mechanics Faculty of Science, University of Yaoundé, P. O. Box 812, Yaoundé, Cameroon \\ Received: 27 January 2015 / Revised: 06 March 2015 / Accepted: 09 March 2015 \\ (C) The author(s) 2015. This article is published with open access at Springerlink.com
}

\begin{abstract}
Computer simulations of friction between polymer brushes are usually simplified compared to real systems in terms of solvents and geometry. In most simulations, the solvent is only implicit with infinite compressibility and zero inertia. In addition, the model geometries are parallel walls rather than curved or rough as in reality. In this work, we study the effects of these approximations and more generally the relevance of solvation on dissipation in polymer-brush systems by comparing simulations based on different solvation schemes. We find that the rate dependence of the energy loss during the collision of brush-bearing asperities can be different for explicit and implicit solvent. Moreover, the non-Newtonian rate dependences differ noticeably between normal and transverse motion, i.e., between head-on and off-center asperity collisions. Lastly, when the two opposing brushes are made immiscible, the friction is dramatically reduced compared to an undersaturated miscible polymer-brush system, irrespective of the sliding direction.
\end{abstract}

Keywords: polymer brushes; biomimetic materials; molecular dynamics simulation

\section{Introduction}

Polymer brushes consist of macromolecules that are attached by one end to surfaces or interfaces at a high density such that the polymer-grafts stretch in the direction normal to the tethering plane [1]. Over the last decades, many applications have been devised using such polymer brushes [2]. They are employed, for example to stabilize colloidal suspensions [3], in oil recovery [4], for protein analysis [5], as anti-fouling coatings [6,7] and as "smart" responsive systems [8], such as drug-delivery systems [9], nano sensors[10, 11] and "pick-up and place" systems [12]. Especially promising is the utilisation of polymer brushes in a biomimetic approach as low-friction surface coatings [13-20], e.g., in artificial joints [21] or industrial applications [22].

* Corresponding author: Martin H. MÜSER.

E-mail: m.mueser@fz-juelich.de
In biological systems, such as human joints, the friction coefficient is very low: It is less than 0.02 [23] even at local pressures up to 50 atmospheres [24]. One of the reasons for this is that sugar chains in the synovial fluid attach to cartilage tissue and protein backbones [25]. The hydrophilic sugar chains keep a water-based, low-viscosity liquid in the joint cavity, resulting in low friction upon relative sliding motion. When polymer brushes are kept in good solvents, the polymers stretch upward [26,27] and, in a similar fashion as in joint lubricants, keep the solvent in the brush on condition that the potentially applied pressure is lower than the osmotic pressure in the solvent [28]. Therefore, polymer brushes are actively studied in the effort to develop biomimetic lubricants.

Computer simulations have been very helpful in providing a better understanding of polymer brushes and the origin of friction is these systems [29]. Early 
molecular dynamics (MD) simulations, which allow for visualizing the positions of polymers, exposed that the macromolecules of opposing brushes interdigitate [30]. Moreover, the inter-brush overlap was observed to correlate with the frictional response of opposing brushes [31,32]. The shear stress on the interpenetrated polymers tilts them such that the overlap zone is reduced with increasing velocity. Therefore, polymer brushes in relative sliding motion can show shear-thinning [31, 32]. Computer simulations of generic, bead-spring models for polymer brushes found that the dissipation in these shear-thinning systems can be described by a sub-linear frictionvelocity relation of $F \sim v^{\kappa}[33,34]$, with $\kappa=0.54-$ 0.57 [33-35]. Experiments revealed that interdigitation can result in high friction $[36,37]$ and cause wear due to chain pull-out and scission [13, 38].

Recent simulations [39] suggested that studies of polymer brushes in simplified geometries, in particular the regularly employed parallel-plate geometry, only see part of what is responsible for dissipation. Engineering surfaces and the exterior of joint implants are rough $[21,40]$ so that new modes of motion and thus dissipation mechanisms can occur in addition to those taking place in a parallel-plate geometry. Firstly, transient interdigitation [41] during an asperity collision must be expected to alter the effective contribution of brush-overlap to the dissipation. Secondly, brushes on curved surfaces deform as they slide past each other, which leads to a viscoelastic hysteresis, which is well known from rubber friction $[42,43]$. Thirdly, when the brushes are deformed, the solvent is partly squeezed out and needs to be re-absorbed. This also causes viscous dissipation [44], which is believed to dominate the friction upon normal approach $[45,46]$ in completely solvated systems. Finally, when different brush-covered asperities move past each other, contacts are formed and broken. This can result in capillary hysteresis, especially in applications where the system is not fully saturated with solvent. All these dissipation mechanisms affect each other and the resulting frictional response is a composition of the different mechanisms [39].

In the last years, several methods have been developed to reduce [47] or even prevent [15] interdigitation of the polymers in opposing brushes. In one method [47], a modulated electric field is applied to tune the degree of overlap between polyelectrolyte brushes. In another method [15], the opposing brushes are chemically distinct such that each brush has its own preferred solvent, e.g., one hydrophilic and one hydrophobic brush, which are immiscible and thus do not interpenetrate. There is only a thin effective overlap zone due to long-wavelength thermal fluctuations of the interface [15]. Consequently, the friction in these immiscible systems can be more than two orders of magnitude lower than the friction for traditional, miscible systems [15]. Due to slip at the interface [48], the effect of the method described above is enhanced when the two solvents are immiscible, but the method can also work when the solvents are miscible, provided that the solvents demix in the contact [49]. Additionally, in a similar fashion as for contacting star polymers [50], it is found that surface curvature can reduce brush interdigitation compared to parallel-plate geometries [49], because polymers can circumvent the effectively-repulsive interaction with other polymers by moving into the preferred good solvent outside the contact.

In this paper, we report non-equilibrium MD simulations of two colliding cylinders that are decorated with polymer brushes. We study how interdigitation and capillaries affect the energy dissipated during an asperity collision. Towards this end, we set up three systems that are each solvated differently. In system 1 , the brushes are completely immersed in implicit solvent. In this system, polymers can escape interdigitation in the $y$ direction (Fig. 1). In system 2, the brushes are undersaturated in explicit solvent such that a capillary forms in the contact. The surface tension of the solvent bundles the polymers together such that the effect of interdigitation on the dissipation in this system is strongly enhanced [49]. System 3 consists of two immiscible polymer brushes, where preferred absorbance of two immiscible solvents in the two chemically different brushes prevents interdigitation of the macromolecules of the opposing brushes. By moving the cylinders in the $y$-direction (Fig. 1) we mimic offcentre collisions between asperities of rough surfaces, while for motion in the normal direction (z-direction, Fig. 1) we mimic head-on asperity collisions. We note that real engineering surfaces do not consist of periodic cylinders, but instead have a roughness distribution over many length scales [51]. Since the relative impor- 
tance of the various dissipation mechanisms depends on many different dimensionless variables [39, 52], it is unlikely that our system precisely mimics the mix of dissipation mechanism in a particular brush system. The analysis of our generic model yet allows one to deepen the understanding of how solvation affects qualitatively interdigitation, capillaries, and energy dissipation during asperity collisions.

\section{Model and methods}

The polymers and the solvent molecules in our simulations are represented by the Kremer-Grest model [53], which is known to qualitatively describe the static and dynamic properties of end-anchored polymers [29], surface-adsorbed molecules [54], polymer melts [55] and polymers in solvent-mixtures [56]. In the Kremer-Grest model, chemically bonded entities are connected via finitely extendable nonlinear elastic (FENE) springs imposed by the potential,

$$
V_{\mathrm{FENE}}=\frac{1}{2} k R_{0}^{2} \ln \left[1-\left(\frac{r_{i j}}{R_{0}}\right)^{2}\right]
$$

with a stiffness of $k=30 \epsilon / \sigma^{2}$ and a maximum extension of $R_{0}=1.5 \sigma$. Here, the parameters $\epsilon$ and $\sigma$ are used to define the units for energy and length, respectively. Typical values are $\epsilon=30 \mathrm{meV}$ and $\sigma=0.5 \mathrm{~nm}$ [57]. Thus, our unit for pressure is $[p]=\epsilon / \sigma^{3} \approx 40 \mathrm{MPa}$. Short-range repulsion and long-range attractive van der Waals interactions are modeled by the Lennard Jones (LJ) potential with the functional form

$$
V_{L J}\left(r_{i j}\right)=4 \epsilon_{i j}\left[\left(\frac{\sigma_{i j}}{r_{i j}}\right)^{12}-\left(\frac{\sigma_{i j}}{r_{i j}}\right)^{6}\right]+V_{\mathrm{c} i j}
$$

for $r_{i j}<r_{\mathrm{c} i j}$, where $r_{i j}$ is the distance between two beads and $r_{\text {cij }}$ is a cutoff beyond which the energy is set to zero. The constant $V_{\text {cij }}$ is chosen so that the potential is continuous at the cutoff. When chosing $r_{c i j}=2^{1 / 6} \sigma_{i j}$, the LJ interaction is purely repulsive, while it contains a significant adhesive tail for $r_{\mathrm{c} i j}=$ $2.5 \sigma_{i j}$. Unless mentioned otherwise, $\sigma_{i j}=\sigma$ and $\epsilon_{i j}=\epsilon$. The LJ interaction between "bonded" beads is always purely repulsive, while that between any other entities needs to be defined for each application. The beads represent Kuhn units. The Kuhn length is generally the length of 3-5 monomers. Thus, the unit of mass can be chosen to be $[\mathrm{m}]=10^{-22} \mathrm{~kg}$ so that the unit of velocity becomes $[v] \approx 7 \mathrm{~m} / \mathrm{s}$.

Figure 1 shows the simulation cells of the three systems studied in this article. They consist of two cut-off cylinders (radius $R=100 \sigma$ and height $h=35 \sigma$ ), which are built up of a single layer of surface-atoms
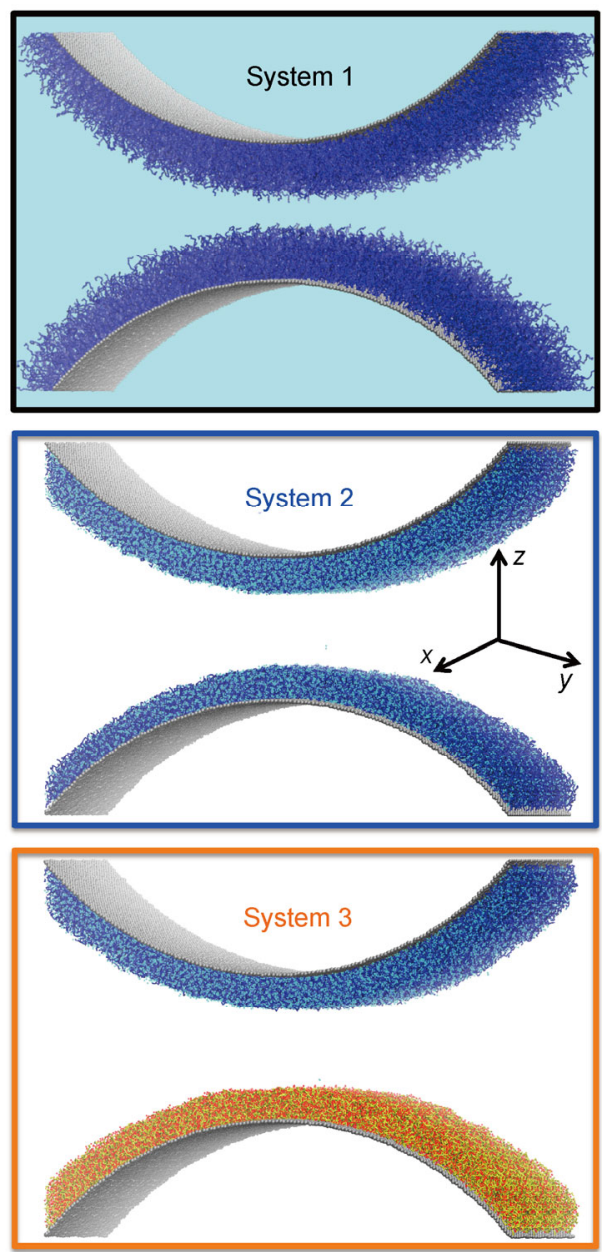

Fig. 1 Snapshots of the three types of setups used in this study. Each time, the system consists of two polymer-bearing cylinders. In system 1, the brushes (dark blue) are physically and chemically the same and completely immersed in an implicit solvent(light blue). In system 2, the brushes (dark blue) are again physically and chemically the same, but undersaturated in explicit solvent (light blue), which is in equilibrium with its gas-phase. In system 3 , the two brushes have identical physical but different chemical properties. Brush 1 (blue) prefers solvent 1 (light blue) and brush 2 (yellow) prefers solvent 2 (red) such that the polymers of the opposing brushes do not want to interdigitate. The snapshots are rendered using VMD [58]. 
in fcc [111] lattice having a nearest-neighbor spacing of $r_{0}=1.2 \sigma$. Nearest-neighbor wall atoms are connected to each other by nonlinear springs of the functional form

$$
V_{n l}\left(r_{i j}\right)=\frac{\epsilon_{\mathrm{b}}\left(r_{i j}-r_{0}\right)^{2}}{\lambda^{2}-\left(r_{i j}-r_{0}\right)^{2}}
$$

with $\epsilon_{\mathrm{b}}=10.3 \epsilon$ and a maximum extension of the spring of $\lambda=0.4 \sigma$. In addition, the wall atoms are connected to their lattice sites with harmonic springs ( $k_{\mathrm{wl}}=32 \epsilon / \sigma^{2}$ ) to prevent drift. On each surface we graft 15,048 polymers of degree of polymerization $N=30$ beads (test runs using $N=100$ beads showed qualitatively similar results). The grafting density $\alpha$ is approximately 2.2 times the critical grafting density $\alpha_{\mathrm{c}}$ above which brushes form [26, 27]. We chose a relatively large interaction length of $\sigma_{\mathrm{bw}}=1.6 \sigma$ for the interaction between individual beads and wall-atoms. This choice together with elastic coupling between wall atoms prevents polymers or solvent molecules from penetrating into the wall. Also, the interaction between the polymers and wall is made repulsive, because attractive interactions can shift $\alpha_{\mathrm{c}}$ to higher values [59], in which case we would need longer or more polymers. The density profiles of brushes on curved surfaces can deviate from those on flat surfaces [60]. Therefore, we made sure that in our system the ratio $N \sigma / R$ is small enough for these effects to become unnoticeable [61].

Periodic boundary conditions are applied in $x$ and $y$ direction. We use the velocity Verlet algorithm, as implemented in LAMMPS [62], to solve Newton's equations of motion. The time step is set to $0.005 \sigma \sqrt{m / \epsilon}$ (test simulations using a timestep of $0.001 \sigma \sqrt{m / \epsilon}$ gave statistically indistinguishable results). The temperature is kept constant at $T=0.6 \epsilon / k_{\mathrm{B}}$. We keep the brushes in relative sliding motion by moving the lattice sites of both surfaces in opposite directions, with constant velocities $\pm v / 2$ in $x, y$, or $z$. To mimic the effect of asperity collision in a multi-asperity contact we chose constant separation over constant normal pressure.

In system 1 (Fig. 1) the two brushes, which are chemically and physically alike, are fully solvated in an implicit solvent. The thermodynamic effect of the implicit solvent is realized by a short-range cutoff for the LJ interaction, which induces an effective repulsion between beads as they experience it in a good solvent. The effect of the solvent viscosity is approximated by a dissipative particle dynamics (DPD) thermostat [63] using a time-constant $\tau=1.0 \sigma \sqrt{m / \epsilon}$. Such a model captures qualitatively the solvent-induced damping and random forces between non-bonded polymer segments while keeping the correct (underdamped) behavior of long-range density fluctuations of sliding polymer brushes in the parallel-plate geometry [64].

In system 2 (Fig. 1) the brushes are also chemically and physically the same, but undersaturated in explicit solvent. For every polymer bead in the brush, there is one solvent bead. Our explicit solvent consists of dimers, because single monomers can induce undesired layering close to walls [33]. The interactions between any two non-bonded beads are now long ranged, specifically $r_{\text {cut }}=2.5 \sigma$, and thus adhesive. Only the interactions between polymer beads had a reduced cutoff of $r_{\text {cut }}=1.6 \sigma$, which still implies some adhesion. The LJ energy parameters between non-connected dimer-units deviated from the default value, $\epsilon_{\mathrm{ss}}=0.5 \epsilon$. This reduced value allowed the solvent to remain liquid within the full pressure range from zero to $25 \mathrm{\epsilon} / \sigma^{3}$, which acts locally for the highest loads. In turn, the interaction parameters between solvent and polymer are increased to $\epsilon_{\mathrm{ps}}=1.2 \epsilon$, which results in good solvent conditions and miscibility. We note that due to our choice of interaction parameters, the Flory Huggings parameters $[65,66]$ of systems 1 and 2 are different. This is one reason why our results for the fully solvated (system 1) and undersaturated (systems 2 and 3) simulation setups can only be qualitatively (and not quantitatively) compared. Another reason is that implicit solvent polymer brush systems often show higher friction than systems described with an explicit solvent [64]. Temperature is kept constant using a Langevin thermostat (time constant $\tau=1.0 \sigma \sqrt{m / \epsilon}$ ) which only acts on the wall-atoms normal to shear and shear-gradient direction such that there is no measurable effect of the thermostat on the friction forces [67].

In system 3, we set up an immiscible polymer brush system. The two opposing brushes are chemically different and each prefer their own solvent while being 
mutually insoluble. To create systems 3 , we used the same setup as for system 2, except that opposing polymers $(\mathrm{P}$ and $\overline{\mathrm{P}})$, different solvents $(\mathrm{S}$ and $\mathrm{S})$ and polymers with the non-preferred solvents (P and $\bar{S} / \bar{P}$ and $S$ ) are made incompatible by shifting the cut-off to the potential minimum; $r_{\text {cut }}=2^{1 / 6} \sigma_{i j}$.

\section{Results and discussion}

\subsection{Motion in transverse direction}

We start the discussion of how different solvent methods affect energy dissipation during the collision of brush-bearing asperity in the case of an off-center collision, in which case, the two contacting surfaces are moved in the $y$-direction. Figure 2 shows selected snapshots of the three investigated systems. Differences between them are revealed most clearly when the center-of-masses of the two asperities are on top of each other, that is, for a (reduced) transverse coordinate of $y=L / 2$, where $L$ is the length of the periodically repeated simulation cell in the $y$ direction $(L=200 \sigma)$. In implicit-solvent simulations, i.e., system 1 mimicking fully immersed systems, the brushes overlap but the sliding-induced asymmetry is not obvious to the eye. In explicit-solvent simulations of a partially wetted homogeneous brush pair (system 2), the interdigitation appears reduced and a clear shape asymmetry becomes visible. In contrast, the brushes of system 3 , where immiscibility is induced by the solvent, show a thin depletion zone between the two brushes and no obvious asymmetry. One can also note that brushes collide earlier in the implicit than the explicit-solvent simulation, despite the absence of a capillary inducing a long-range attraction in the latter, undersaturated system. This difference is caused by the solvation method. In system 1, the polymers are free to stretch into the surrounding solvent [49] and the density profiles show the typical gradual decay in polymer density for increasing distances from the surface characteristic for swollen polymer brushes [28, 68-70]. In contrast, in systems 2 and 3, the undersaturation of solvent confines the polymers. In these brushes, the polymer density is higher than that in system 1 and the density profiles resemble a step function with a slightly enhanced solvent density at the surface due to our choice for the interaction parameters [71]. Consequently, the radius of gyration in the direction
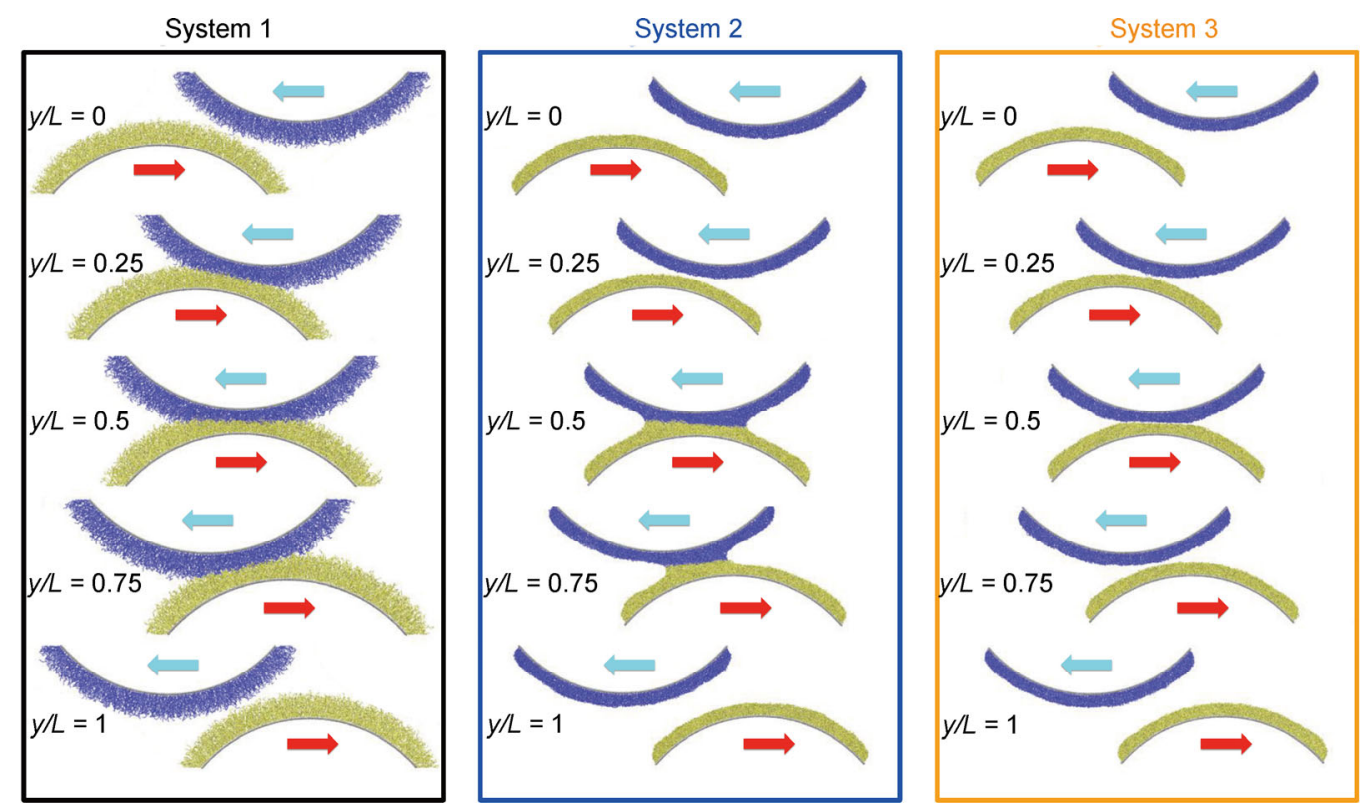

Fig. 2 Snapshots of the three systems upon relative sliding motion $(v=0.01 \sigma / \tau)$ in the transverse direction. The images in the left column show system 1 at $y / L=0,0.25,0.5,0.75$, and 1 from top to bottom. The images in the middle and the right columns show systems 2 and 3, respectively, at the same distances as system 1. The opposing brushes are colored differently for clarity, even when brushes are alike (as in systems 1 and 2). For all systems the distance between the cylinder-apices is $14.6 \sigma$ at $y=L / 2(L$ is the length of the box in $y$ of $L=200 \sigma$ for all systems in these simulations). The snapshots are rendered using VMD [58]. 
normal to the surface is much larger for the solventimmersed brushes in system 1 and thus, contacts are earlier formed compared to systems 2 and 3.

Not only the snapshots but also the instantaneous lateral forces $F_{y}$, or force traces, strongly depend on the solvation method. Figure 3 shows typical force traces for systems 1 (top), 2 (middle) and 3 (bottom) for various velocities $v$ and for $v \rightarrow 0$. To minimize the computational costs, we reduced the box size in $y$ compared to Fig. 2 for systems 2 and 3 to $L=168 \sigma$ and $L=158 \sigma$, respectively. We remind the reader that $y=L / 2$ is a symmetry point for an equilibrium system, that is, for a system moving at infinitely small velocity. This symmetry implies that the free energy is a symmetric function with respect to $y=L / 2$, so that its derivative, the equilibrium force indicated by an orange line, is asymmetric with respect to that point. To obtain the forces for $v \rightarrow 0$ for systems 1 and 3 (Fig. 3, orange lines), we first projected out the ( $v \rightarrow 0$-forbidden) symmetric contributions to the force trace by calculating $F_{\text {as }}(y / L, v)=\left(F_{y}(y / L, v)-F_{y}(1-\right.$ $y / L, v)) / 2$ for each $y / L$ and for each velocity $v$. Next, the forces $F_{0}(y / L)$ at $v \rightarrow 0$ were obtained by fitting the anti-symmetrized forces $F_{\text {as }}(y / L, v)$ according to $F_{\text {as }}(y / L, v)=F_{0}(y / L)+$ const $v^{\kappa}$. This procedure was not sufficient for system 2 , because the deviation between finite-velocity and equilibrium friction traces could not be represented by a single contribution scaling as a simple power law of velocity.

To obtain the forces for $v \rightarrow 0$ for system 2 (Fig. 3, orange line), we positioned the cylinders at fixed equilibrium distances $y / L$ and monitored the evolution of $F_{y}(y / L)$. The forces as a function of time were each fitted with the function $F_{y}(y / L, t)=F_{0}(y / L)+C e^{-t / \tau}$, where $C$ and $\tau$ are adjustable parameters describing the decay to the fitting parameter $F_{0}(y / L)$ that we used as an estimate for the position-dependent force at $v \rightarrow 0$. Next, the force $F_{0}(y / L)$ was fitted on the domain $0.2 \leqslant y / L<1$ with

$$
F_{0}=A_{1} \sin \left\{\frac{2 \pi}{L^{\prime}}(y-L / 2)\right\}+A_{2} \sin \left\{\frac{4 \pi}{L^{\prime}}(y-L / 2)\right\}
$$

where $A_{1}$ and $A_{2}$ are fitting parameters and $L^{\prime}$ the length over which a given capillary does not become unstable. Thus, $F_{0}$ does not represent the mean lateral force in full equilibrium but only in a "restricted"
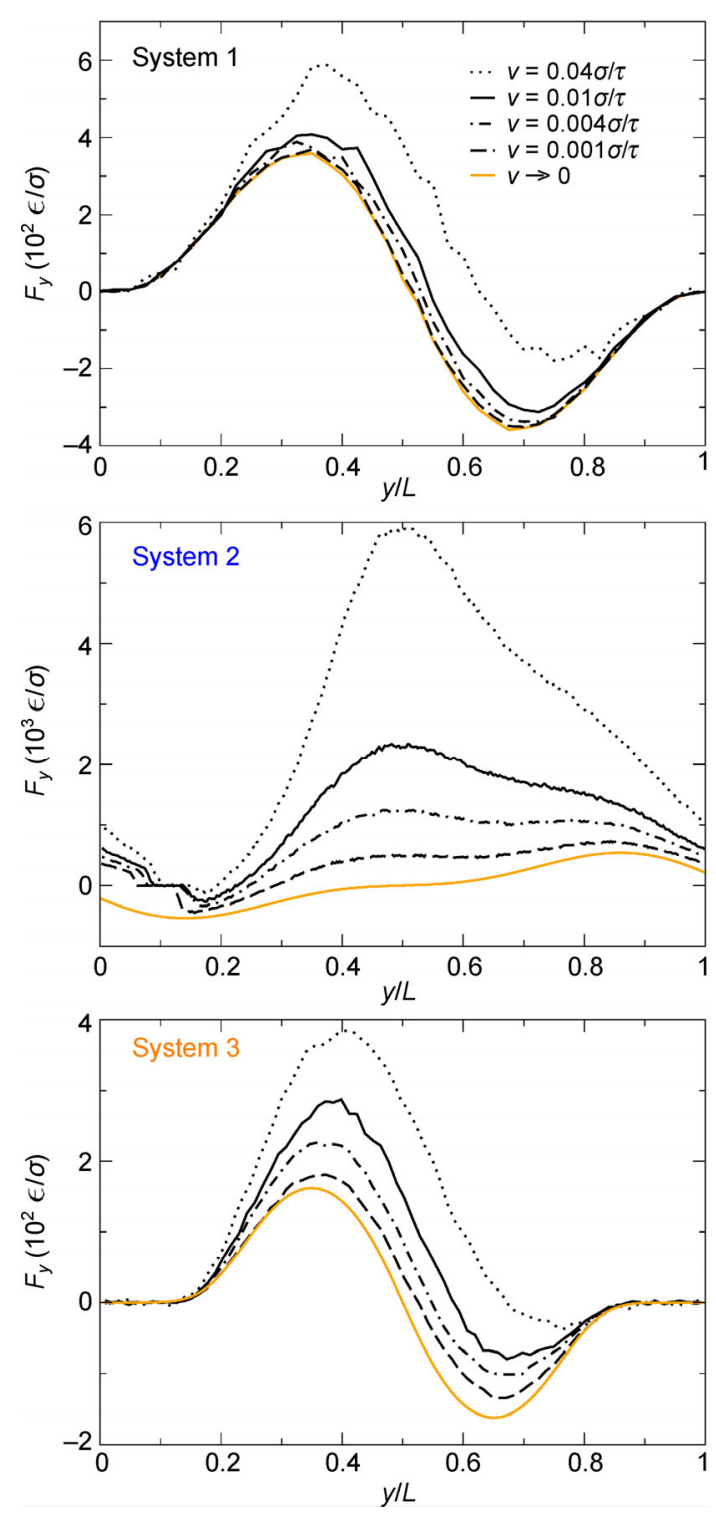

Fig. 3 Force $F_{y}$ traces for cylinders sliding at constant height in the transverse direction at various relative velocities $v$ for system 1 (top), system 2 (middle) and system 3 (bottom). $L$ is the length of the box in $y, L=200 \sigma$ for system $1, L=168 \sigma$ for system 2 and $L=158 \sigma$ for system 3 . The orange line indicates the mean force for $v \rightarrow 0$. For system 2 , the mean force is calculated in a restricted equilibrium, in which the top asperity forms a capillary with the bottom asperity located at $y=L / 2$. The corresponding orange line is solid where the capillary is mono-stable and dotted where a broken capillary is (meta-)stable.

equilibrium, in which the top asperity forms a capillary with the bottom asperity centered at $y=L / 2$. Since the mean lateral force in this restricted equilibrium goes to zero at $y=L / 2 \pm L^{\prime} / 2$, we can use a sine expansion, which we truncate after two terms in Eq. 4. 
The latter parameter turned out to slightly exceed $L$ so that our fit implicitly includes higher-order harmonics, albeit at the expense of being discontinuous at the periodic boundary. The fit to this function is the orange line in Fig. 3 for system 2.

When comparing the force traces at finite velocities for the different systems, it becomes clear that the shape of the force traces of systems 1 and 3 are quite similar, while that of system 2 is distinctively different. Since the main difference between system 2 and systems 1 and 3 is the absence of capillary hysteresis in the latter, the qualitative difference in shape of the force traces for system 2 is determined by contactformation and -break-up during sliding. Another distinct difference is the stochastic noise observed in system 1 compared to systems 2 and 3 . In system 1 , the polymers are free to move into the solvent resulting in much larger interfacial fluctuations than that in system 2 and 3. This causes larger fluctuations in the temporal force.

After subtracting off the $v \rightarrow 0$ force from the traces of systems 1 and 3, a single peak remains, which is almost symmetric around $y=L / 2$. The height and the width of the peaks depend on the velocity, which we quantified by fitting the traces with a Gaussian:

$$
F_{0}=F_{\text {peak }} \exp \left\{\frac{-(y-L / 2)^{2}}{2 w^{2}}\right\}
$$

where $F_{\text {peak }}$ is the maximum of the peak and $w$ is the Gaussian width of the peak. The dissipation in systems 1 and 3 is caused by different mechanisms. In system 3 only solvent flow (hydrodynamic interactions) and shape hysteresis contributes to the dissipation, while in system 1 also interdigitation can contribute. Since the force traces of both systems can be fitted quite well using Eq. (5), we can conclude that all these dissipation mechanisms induce a single peak with a maximum near the symmetry point $y=L / 2$.

For systems 1 and 3 , both $F_{\text {peak }}$ and $w$ vary with the sliding velocity and can both be fitted with the generic power-law relation $F_{\text {peak }} \sim v^{\kappa}$ or $w \sim v^{\omega}$. Even though the shape of the peak is the same for systems 1 and 3, the velocity dependency of $F_{\text {peak }}$ differs distinctly from that of $w$. For system $1, F_{\text {peak }}$ increases linearly with velocity $(\kappa=1)$, while $w$ decreases very slightly with velocity $(\omega=0.08)$. Only for velocities higher than $v=0.4 \sigma / \tau$ does the non-equilibrium, excess peak height starts to scale sub-linearly with velocity $(\kappa=$ 0.64 ) and the width of the peak starts to decrease more strongly with increasing velocity. For system $3, F_{\text {peak }}$ increases sub-linearly with velocity $(\kappa=0.51)$, while $w$ decreases stronger with velocity $(\omega=0.15)$ than system 1 . The shear-thinning exponents of systems 1 and 3 are different and thus provide an indication of the dominating dissipation mechanisms. But, as discussed before [39], it is difficult to assign shearthinning exponents to different dissipation mechanisms, because the mechanisms are intertwined resulting in an effective exponent that is determined by the relative contribution of the mechanisms.

Figure 4 contains a more in-depth analysis of the asperity collision in system 2 . The finite-velocity force trace is best discussed together with the snapshots shown in Fig. 2. At $y / L \approx 0.1$, the lateral force shown in Fig. 4(a) is equal to zero, implying that the brushes, including their solvation shell, do not touch. Upon sliding, a negative, i.e., attractive force acts between the two brushes, which can be readily interpreted as a capillary attraction. At $y=L / 4$, the lateral force is already positive, although the polymers of opposing brushes do not yet see each other directly. This force results from the solvent not being very compressible while having finite inertia. The excess force $\Delta F=F(v=$ finite $)-F(v=0)$ then shows a prominant maximum and clearly visible shoulder. The maximum is located near the symmetry point $y=L / 2$, which is where the polymers start to interditate as well as to reach a state of high compression. The shoulder (which becomes a clearly visible peak when subtracting the excess force associated with the first peak) lies near $y / L=0.75$, which is where the polymer gets stretched due to the capillary trying to minimize its surface. This latter process is absent in systems 1 and 3, which is why they only have one excess peak. Interestingly, the two dissipation processes of system 2 show a similar rather dependence on rate: As one can see in Fig. 4(b), both excess contributions scale proportionally to $v^{0.68 \pm 0.01}$. This degeneracy can be fortuitous but we argue it is more likely that it results from the dissipation mechanisms being strongly intertwined, since in both cases viscoelasticity plays a prominent role. Note that the mean excess force $\{\Delta F\}$, which one can equate 

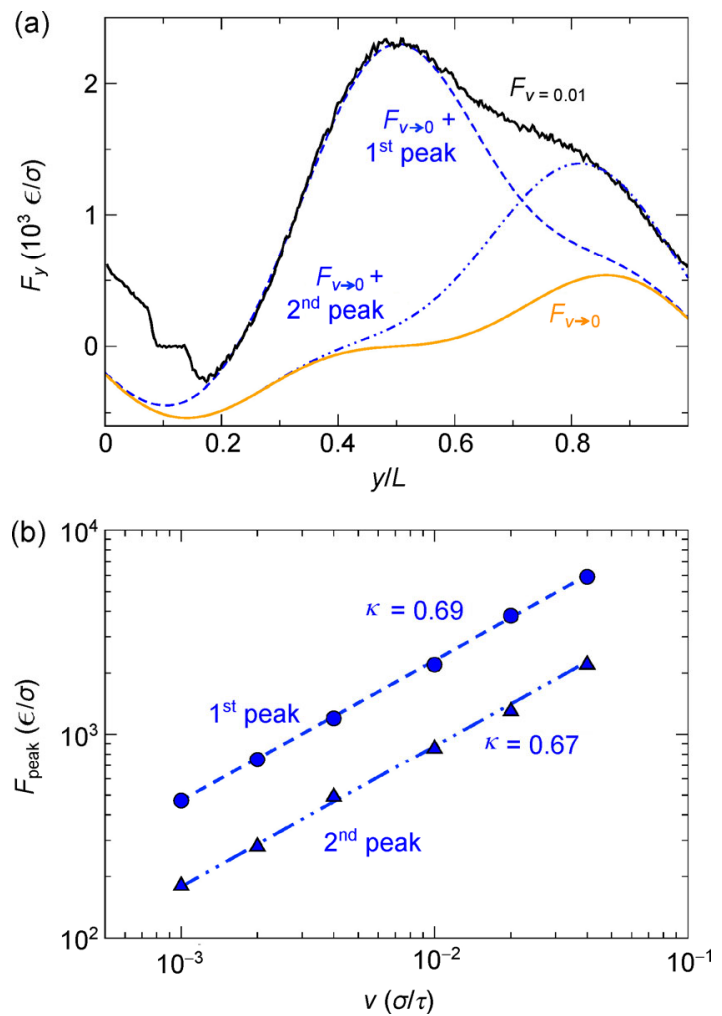

Fig. 4 (a) The total friction force $F_{y}$ (black line) obtained while sliding the cylinders of system 2 at a relative velocity of $v=0.01 \sigma / \tau$ in the transverse direction over one period image ( $L$ denotes the box length of the simulation cell in $y$ ). The blue lines depict the force composed of the force in the limit of $v \rightarrow 0$ (orange line) plus the Gaussian function fitted to the first (dash) or the second (double dot dash) peak in the simulated force trace (black line). (b) The height $F_{\text {peak }}$ of the Gaussian functions fitted to the first peak (circles) and second peak (triangles) versus the sliding velocity $v$. The lines are fits to the power law relation $F_{\text {peak }}=v^{\kappa}$.

with the friction force, can show different scaling than of the two peaks discussed so far, because the capillary formation and break-up process are strongly asymmetric.

A summary of the total energy dissipation during an off-center asperity collision is presented in Fig. 5, which shows the integrated force traces $W=\int_{0}^{L} F_{y}(y) \mathrm{d} y$ of Fig. 3, which related to the mean kinetic friction force by $F_{k}=W / L$. It reveals that the two symmetric brush systems (systems 1 and 2) have different prefactors and show different rate dependences according to $\Delta F \propto v^{\kappa}$ despite having many of the dissipation mechanisms in common. One might argue that we slide at different velocities compared to the critical shearrate for shear-thinning or Weissenberg number

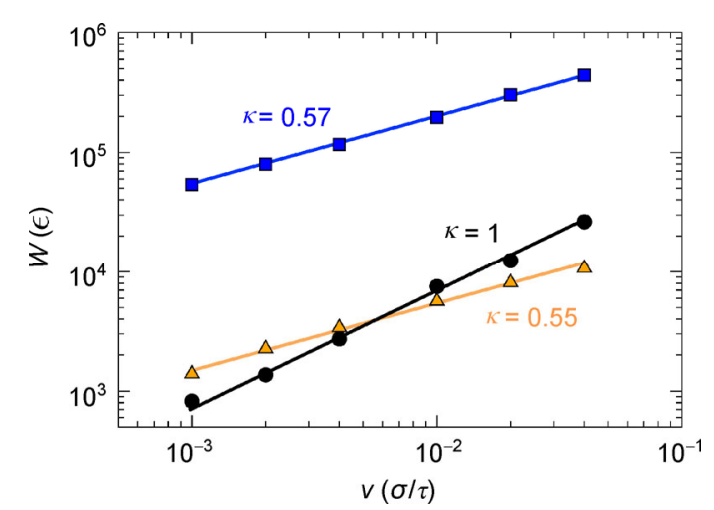

Fig. 5 The work $W$ per asperity collision versus velocity $v$ for system 1 (black circles), system 2 (blue squares) and system 3 (orange triangles). The solid lines are fits to the power law relation $W=v^{\kappa}$

for interdigitation as the dominant cause of friction. However, system 2 shows a larger exponent of $\kappa=0.67$ also outside linear response. In contrast to explicit solvent simulations [39], the shear-thinning exponent in these implicit solvent simulations is found to be independent of the viscosity (damping-coefficient of the DPD thermostat). This implies that a different dissipation mechanism is dominating the friction for asperity collisions simulated using implicit solvent compared to explicit solvent. The lower exponent for system 2 is most likely caused by capillary hysteresis in combination with the different mix of intertwined dissipation mechanisms. The dissipated energy in the mutually insoluble brushes (system 3) display a similar shear thinning exponent as system 1 , though these asymmetric systems have much reduced prefactor of the average friction.

\subsection{Motion in normal direction}

We repeated our rate-dependence analysis of dissipated energy per asperity collision for head-on collisions, i.e., for motion in the direction normal to the (mean) surface director. Corresponding snapshots are presented in Fig. 6. Differences between different solvation methods can be detected, although differences are smaller than that for the off-center collisions. The interdigitation is largest in the implicit-solvent simulations, the reason being again that the implicit solvent only adds some damping but does not need to get squeezed out during the asperity collision. An adhesive neck is only present in the understaturated, explicitsolvent simulations, while overlap between the brushes 

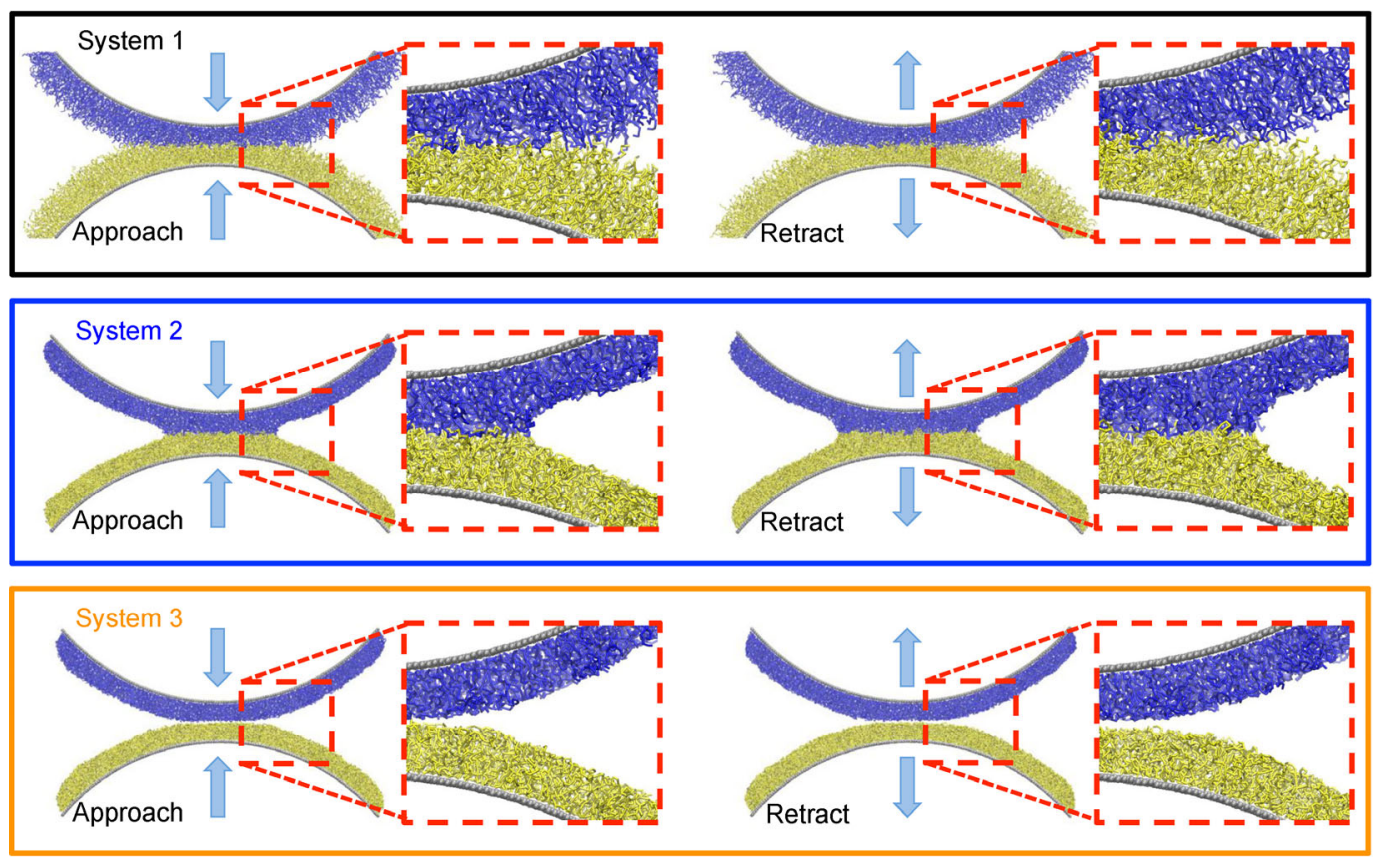

Fig. 6 Snapshots of the three systems upon relative motion $(v=0.01 \sigma / \tau)$ in the normal direction at $y=L / 2$ and $\Delta z=z-z_{0}=2.5 \sigma$, where $z_{0}$ is the distance of velocity-inversion, which we chose to be at a distance of $14.6 \sigma$ between the cylinder-apices. The opposing brushes are colored differently for clarity, even when brushes are chemically alike, as in systems 1 and 2 . The images on the left show the contact at approach and the images on the right show the contact upon retract. The snapshots are rendered using VMD [58].

is only avoided in the mutually insoluble brushes with explicit solvent.

Force traces corresponding to the figure discussed in the precedent paragraph are shown in Fig. 6. They are again sensitive to the solvation method. In particular, the hystersis is strongest for the undersaturated, explicit-solvent miscible brush simulation (system 2), which shows a jump-into-contact instability and very large pull-off forces when moving the two brushes out of contact again. The implicit-solvent, fully saturated system (system 1 ) shows practically no visible hysteresis. In fact, at the given level of approximation (generic bead-spring models), it is so small that it proved difficult to obtain meaningful values for the dissipated energy per compression cycle, which would clearly exceed the stochastic noise. The (unavoidable) hysteresis for the asymmetric system 3 is much smaller than for the miscible, explicit solvent system 2 . It is nevertheless larger than the hysteresis in the implicit-solvent simulations of system 1 . The latter can be expected, since the dissipation in the normal direction results from the squeeze out of explicit solvent, which is not realistically captured by a DPD thermostat. Moreover, the increased polymer-density in the undersaturated immiscible system enhances the drag force on the solvent.

The area between compression and decompression traces in Fig. 7 give the energy dissipated during a (head-on) asperity collision (Fig. 8). As for the off-center collision, solvent-induced miscible and immiscible polymer brush systems reveal similar scaling for the head-on collisions. The pertinent exponent differs from the previous one, i.e., this time the lost energy

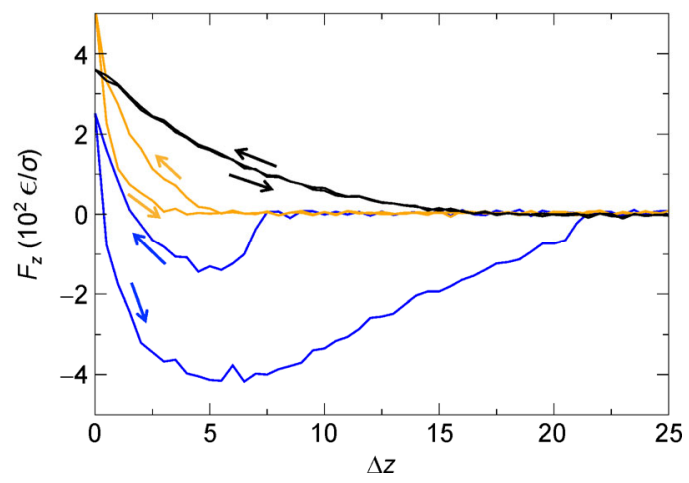

Fig. 7 Force $F_{z}$ versus $\Delta z=z-z_{0}$ upon moving the cylinders in the normal direction at $v=0.01 \sigma / \tau$ for system 1 (black), system 2 (blue) and system 3 (orange). For all systems the minimum distance between the cylinder-apices is $14.6 \sigma$, which we chose as $z_{0}$. 


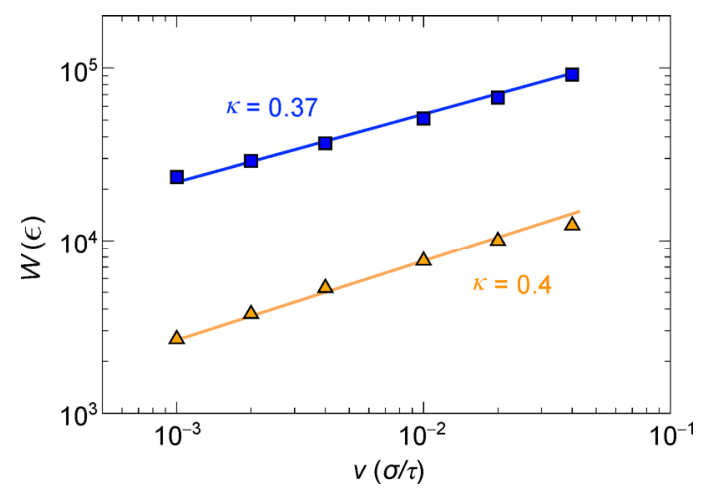

Fig. 8 The work $W$ per indentation-cycle in the normal direction versus velocity $v$ for system 2 (blue squares) and system 3 (orange triangles). The solid lines are fits to the power law relation $W \propto \operatorname{const} v^{\kappa}$.

scales according to $v^{0.385 \pm 0.015}$. Seeing different exponents for rate dependence in normal and transverse direction is not surprising [72-74], because even simple fluids in linear response show a direction-dependent effective in the presence of a (symmetry-breaking) wall $[75,76]$.

\section{Conclusions}

In this work, we scrutinized how different solvation methods affect the dissipated energy when two polymer-brush decorated asperities collide into each other. We found that different factors, which are often dealt with half-heartedly in simulations of pertinant systems such as the parallel plate geometry, can affect not only the prefactors but also the functional form of how dissipated energy depends on shear or compression/decompression rate. Because the overall dissipation (prefactors and shear-thinning exponents) is determined by the mix of dissipation mechanisms, the exact solvation-method strongly affects the frictional response to relative motion. Moreover, (shear-thinning) exponents differ between normal and transverse motion. Decomposing the direction-dependent shear thinning into the exact relative contribution of the different dissipation mechanisms requires further research.

\section{Acknowledgements}

We thank the Jülich Supercomputing Centre for computation time. SdB acknowledges financial support by the Foundation for Fundamental research on Matter
(FOM), which is financially supported by the Netherlands Organization for Scientific Research (NWO). GDK thanks Twas-DFG Cooperation Visits Programme for financial support.

\section{Appendix}

PACS: 47.57.Ng Polymers and polymer solutions; 87.85.gf Fluid mechanics and rheology; 87.85.gp Mechanical systems

Open Access: This article is distributed under the terms of the Creative Commons Attribution License which permits any use, distribution, and reproduction in any medium, provided the original author(s) and source are credited.

\section{References}

[1] Milner S T. Polymer brushes. Science 251: 905-914 (1991)

[2] Azzaroni, O. Polymer brushes here, there, and everywhere: Recent advances in their practical applications and emerging opportunities in multiple research fields. J Polym Sci Part A: Polym Chem 50: 3225-3258 (2012)

[3] van der Waarden M. Stabilisation of carbon-black dispersions in hydrocarbons. J Colloid Sci 5: 317-325 (1950)

[4] Stanislav J F. Use of polymers in oil recovery processes. In Proceedings of the First Conference of European Rheologists, Graz, Austria, 1982: 210-211.

[5] Jain P, Baker G L, Bruening M L. Applications of polymer brushes in protein analysis and purification. Annu Rev Anal Chem 2: 387-408 (2009)

[6] Chang Y, Liao S-C, Higuchi A, Ruaan R-C, Chu C-W, Chen W-Y. A highly stable nonbiofouling surface with well-packed grafted zwitterionic polysurfobetaine for plasma protein repulsion. Langmuir 24: 5453-5458 (2008)

[7] Wattendorf U, Merkle H P. PEGylation as a tool for biomedical engineering of surface modified particles. $J$ Pharm Sci 97: 4655-4669 (2008)

[8] Cohen Stuart M A, Huck W T S, Genzer J, Müller M, Ober C, Stamm M, Sukhorukov G B, Szleifer I, Tsukruk V V, Urban M, Winnik F, Zauscher S, Luzinov I, Minko S. Emerging applications of stimuli-responsive polymer materials. Nature Mater 9: 101-113 (2010)

[9] Bajpai A K, Shukla S K, Bhanu S, Kankane S. Responsive polymers in controlled drug delivery. Prog Polym Sci 33: 1088-1118 (2008)

[10] Merlitz H, He G-L, Wu C-X, Sommer J-U. Nanoscale 
brushes: How to build a smart surface coating. Phys Rev Lett 102: 115702 (2009)

[11] Tokareva I, Minko S, Fendler J H, Hutter E. Nanosensors based on responsive polymer brushes and gold nanoparticle enhanced transmission surface plasmon resonance spectroscopy. J Am Chem Soc 126: 15950-15951 (2004)

[12] Yu Y, Kieviet B D, Kutnyanszky E, Vancso G J, de Beer S. Cosolvency-induced switching of the adhesion between poly(methyl methacrylate) brushes. ACS Macro Lett 4: 75-79 (2015)

[13] Bureau L, Léger L. Sliding friction at a rubber/brush interface. Langmuir 20: 4523-4529 (2004)

[14] Chen M, Briscoe W H, Armes S P, Klein J. Lubrication at physiological pressures by polyzwitterionic brushes. Science 323: 1698-1701 (2009)

[15] de Beer S, Kutnyanszky E, Schön P M, Vancso G J, Müser M H. Solvent induced immiscibility of polymer brushes eliminates dissipation channels. Nat Commun 5: 3781 (2014)

[16] Klein J, Kumacheva E, Mahalu D, Perahia D, Fetters L J. Reduction of frictional forces between solid surfaces bearing polymer brushes. Nature 370: 634-636 (1994)

[17] Lee S, Spencer N D. Aqueous lubrication of polymers: Influence of surface modification. Tribol Int 38: 922-930 (2005)

[18] Li A, Benetti E M, Tranchida D, Clasohm J N, Schönherr H, Spencer N D. Surface-grafted, covalently cross-linked hydrogel brushes with tunable interfacial and bulk properties. Macromolecules 44: 5344-5351 (2011)

[19] O'Shea S J, Welland M E, Rayment T. An atomic force microscope study of grafted polymers on mica. Langmuir $\mathbf{9}$ : 1826-1835 (1993)

[20] Sui X, Zapotoczny S, Benetti E M, Schön P, Vancso G J. Characterization and molecular engineering of surface-grafted polymer brushes across the length scales by atomic force microscopy. J Mater Chem 20: 4981-4993 (2010)

[21] Moro T, Takatori Y, Ishihara K, Konno T, Takigawa Y, Matsushita T, Chung U-I, Nakamura K, Kawaguchi H. Surface grafting of artificial joints with a biocompatible polymer for preventing periprosthetic osteolysis. Nature Mater 3: 829-836 (2004)

[22] Lee S, Spencer N D. Sweet, hairy, soft, and slippery. Science 319: 575-576 (2008)

[23] Jones E. Joint lubrication. Lancet 223: 1426 (1934)

[24] McCutchen $\mathrm{C} \mathrm{W}$. The frictional properties of animal joints. Wear 5: 1-17 (1962)

[25] Wilkins J. Proteolytic destruction of synovial boundary lubrication. Nature 219: 1050-1051 (1968)

[26] Alexander S. Adsorption of chain molecules with a polar head a scaling description. J Phys-Paris 38(8): 983-987 (1977)
[27] de Gennes P G. Conformations of polymers attached to an interface. Macromolecules 13: 1069-1075 (1980)

[28] Milner S T, Witten T A, Cates M E. Theory of the grafted polymer brush. Macromolecules 21: 2610-2619 (1988)

[29] Binder K, Kreer T, Milchev A. Polymer brushes under flow and in other out-of-equilibrium conditions. Soft Matter 7: 7159-7172 (2011)

[30] Murat M, Grest G S. Interaction between grafted polymeric brushes: A molecular-dynamics study. Phys Rev Lett 63: 1074-1077 (1989)

[31] Grest G S. Interfacial sliding of polymer brushes: A molecular dynamics simulation. Phys Rev Lett 76: 4979-4982 (1996)

[32] Kreer T, Müser M H, Binder K, Klein J. Frictional drag mechanisms between polymer-bearing surfaces. Langmuir 17: 7804-7813 (2001)

[33] Galuschko A, Spirin L, Kreer T, Johner A, Pastorino C, Wittmer J, Baschnagel J. Frictional forces between strongly compressed, nonentangled polymer brushes: Molecular dynamics simulations and scaling theory. Langmuir 26: 6418-6429 (2010)

[34] Spirin L, Galuschko A, Kreer T, Johner A, Baschnagel J, Binder K. Polymer brush lubrication in the limit of strong compression. Eur Phys J E 33: 307-311 (2010)

[35] de Beer S. Switchable friction using contacts of stimulusresponsive and nonresponding swollen polymer brushes. Langmuir 30: 8085-8090 (2014)

[36] Klein J. Shear, friction and lubrication forces between polymerbearing surfaces. Annu Rev Mater Sci 26: 581-612 (1996)

[37] Léger L, Raphaël E, Hervet H. Surface-anchored polymer chains: Their role in adhesion and friction. Adv Polym Sci 138: 185-225 (1999)

[38] Maeda N, Chen N, Tirrell M, Israelachvili, J N. Adhesion and friction mechanisms of polymer-on-polymer surfaces. Science 297: 379-382 (2002)

[39] de Beer S, Müser M H. Alternative dissipation mechanisms and the effect of the solvent in friction between polymer brushes on rough surfaces. Soft Matter 9: 7234-7241 (2013)

[40] Wang N, Trunfio-Sfarghiu A-M, Portinha D, Descartes S, Fleury E, Berthier Y, Rieu J-P. Nanomechanical and tribological characterization of the MPC phospholipid polymer photografted onto rough polyethylene implants. Colloids Surf B 108: 285-294 (2013)

[41] Briels W J. Transient forces in owing soft matter. Soft Matter 5: 4401-4411 (2009)

[42] Persson B N J. Theory of rubber friction and contact mechanics. J Phys Chem 115: 3840 (2001)

[43] Tabor, D. Hysteresis losses in the friction of lubricated rubber. Rubber Chem Technol 33: 142-150 (1958)

[44] Nommensen P A, Duits M H G, van den Ende D, Mellema J. 
Steady shear behavior of polymerically stabilized suspensions: Experiments and lubrication based modeling. Phys Rev E 59: 3147-3154 (1999)

[45] Balko S M, Kreer T, Costanzo P J, Patten T E, Johner A, Kuhle T L, Marques C M. Polymer brushes under high load. PLoS ONE 8: e58392 (2013)

[46] Klein J, Kamiyama Y, Yoshizawa H, Israelachvili J N, Fredrickson G H, Pincus P, Fetters L J. Lubrication forces between surfaces bearing polymer brushes. Macromolecules 26: 5552-5560 (1993)

[47] Drummond C. Electric-field-induced friction reduction and control. Phys Rev Lett 109: 154302 (2012)

[48] Koplik J, Banavar J R. Slip, immiscibility and boundary conditions at the liquid-liquid interface. Phys Rev Lett 96: 044505 (2006)

[49] de Beer S, Müser M H. Friction in (im-) miscible polymer brush systems and the role of transverse polymer tilting. Macromolecules 47: 7666-7673 (2014)

[50] Lo Verso F, Yelash L, Egorov S A, Binder K. Interactions between polymer brush-coated spherical nanoparticles: The good solvent case. J Chem Phys 135: 214902 (2011)

[51] Persson B N J, Albohr O, Tartaglino U, Volokitin A I, Tosatti E. On the nature of surface roughness with application to contact mechanics, sealing, rubber friction and adhesion. $J$ Phys: Condens Matt 17: R1-R62 (2005)

[52] Kong L-T, Denniston C, Müser M H. The crucial role of chemical detail for slip-boundary conditions: Molecular dynamics simulations of linear oligomers between sliding aluminum surfaces. Modelling Simul Mater Sci Eng 18: 034004 (2010)

[53] Grest G S, Kremer K. Molecular dynamics simulation for polymers in the presence of a heat bath. Phys Rev A 33: 3628 (1986)

[54] He G, Müser M H, Robbins M O. Adsorbed layers and the origin of static friction. Science 284: 1650-1652 (1999)

[55] Priezjev N V, Troian S M. Molecular origin and dynamic behavior of slip in sheared polymer films. Phys Rev Lett 92: 018302 (2004)

[56] Mukherji D, Marques C M, Kremer K. Polymer collapse in miscible good solvents is a generic phenomenon driven by preferential adsorption. Nat Commun 5: 4882 (2014)

[57] Kremer K, Grest G S. Dynamics of entangled linear polymer melts: A molecular-dynamics simulation. J Chem Phys 92: 5057 (1990)

[58] Humphrey W, Dalke A, Schulten K. VMD: Visual molecular dynamics. J Molec Graphics 14: 33-38 (1996)

[59] Descas R, Sommer J-U, Blumen A. Grafted polymer chains interacting with substrates: Computer simulations and scaling.
Macromol Theory Simul 17: 429-453 (2008)

[60] Binder K, Milchev A. Polymer brushes on flat and curved surfaces: How computer simulations can help to test theories and interpret experiments. J Polym Sci Part B: Polym Phys 50: 1515-1555 (2012)

[61] Murat M, Grest G S. Polymers end-grafted onto a cylindrical surface. Macromolecules 24: 704-708 (1991)

[62] Plimpton S. Fast parallel algorithms for short-range molecular dynamics. J Comp Phys 117: 1-19 (1995)

[63] Groot R D, Warren P B. Dissipative particle dynamics: Bridging the gap between atomistic and mesoscopic simulation. J Chem Phys 107: 4423-4435 (1997)

[64] Pastorino C, Kreer T, Müller M, Binder K. Comparison of dissipative particle dynamics and Langevin thermostats for out-of-equilibrium simulations of polymeric systems. Phys Rev E 76: 026706 (2007)

[65] Flory P J. Thermodynamics of high polymer solutions. $J$ Chem Phys 10: 51 (1942)

[66] Huggins, M. L. Ann. N.Y. Thermodynamic properties of solutions of long-chain compounds. Acad Sci 43: 1-32 (1942)

[67] Thompson P A, Robbins M O. Shear flow near solids: Epitaxial order and flow boundary conditions. Phys Rev A 41: 6830 (1990)

[68] Auroy P, Mir Y, Auvray L. Local structure and density profile of polymer brushes. Phys Rev Lett 69: 93-95 (1992)

[69] Lai P-Y, Binder K. Structure and dynamics of grafted polymer layers: A Monte Carlo simulation. J Chem Phys 95: 9288 (1991)

[70] Murat M, Grest G S. Structure of grafted polymeric brushes in solvents of varying quality: A molecular dynamics study. Macromolecules 26: 3108-3117 (1993)

[71] Cohen Stuart M A, de Vos W M, Leermakers F A M. Why surfaces modified by exible polymers often have a finite contact angle for good solvents. Langmuir 22: 1722-1728 (2006)

[72] Dhinojwala A, Cai L, Granick S. Critique of the friction coefficient concept for wet (lubricated) sliding. Langmuir 12: 4537-4542 (1996)

[73] Ringlein, J., and Robbins, M. O. Understanding and illustrating the atomic origins of friction. Am J Phys 72: 884-891 (2004)

[74] Yoshizawa H, Chen Y-L, Israelachvili J N. Fundamental mechanisms of interfacial friction. 1. Relation between adhesion and friction. J Phys Chem 97: 4128-4140 (1993)

[75] Brenner H. The slow motion of a sphere through viscous fluid towards a plane surface. Chem Eng Sci 16: 242-251 (1961)

[76] Schindler M. A numerical test of stress correlations in fluctuating hydrodynamics. Chem Phys 375: 327-336 (2010) 


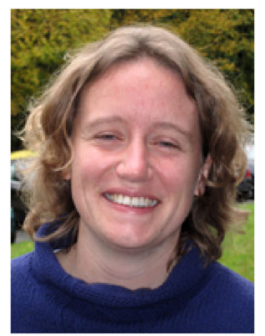

Sissi de BEER. She obtained her PhD degree in the Physics of Complex Fluids Group of the University of Twente, the Netherlands, in 2011. Her PhD thesis describes atomic force microscopy experiments and molecular dynamics (MD) simulations of confined simple liquids. From 2011 till 2013, she worked as a postdoctoral fellow at the Jülich

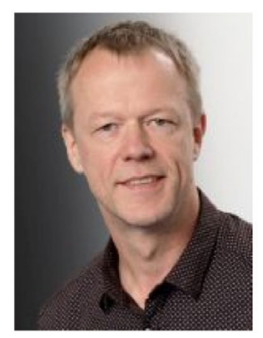

Martin H. MÜSER. He received his diploma in physics from Saarland University, Germany, in 1992 and his Ph.D degree in theoretical physics from Johannes Gutenberg University Mainz in 1995. He spent his postdoctoral time in the Department of Chemistry at Columbia University and in the Physics \& Astronomy Department at the Johns Hopkins
Supercomputing Centre (Forschungszentrum Jülich) focusing on MD simulations to study polymer brush friction. Since November 2013, Sissi is a research associate in the Materials Science and Technology of Polymers Group of the University of Twente, where she now combines simulations with experimental techniques to understand the (tribo-)mechanical response and absorptive properties of polymer brushes in (bio-)technical applications.

University. In 2002, he became a professor of applied mathematics at Western University in London, Ontario. After a sabbatical year at IBM, T. J. Watson, New York, he moved to Saarland University in 2009, where he holds the chair of material simulations in the Department of Material Science and Technology. Since 2011, he also heads the Computational Materials Research Group at the Supercomputing Centre of Forschungszentrum Jülich. 